\title{
Construcción y evidencias de validez de una escala abreviada de autoinforme para evaluar el autoritarismo
}

\author{
ANA CRISTINA ÁVILA-BATISTA \\ Universidade São Francisco, Brasil \\ ORCID: https://orcid.org/0000-0001-5820-5066 \\ FABIÁN J. M. RUEDA* \\ Universidade São Francisco, Brasil \\ ORCID: https://orcid.org/0000-0001-5173-0802 \\ NELSON HAUCK FILHO \\ Universidade São Francisco, Brasil \\ ORCID: https://orcid.org/0000-0003-0121-7079
}

\begin{abstract}
How to quote this article: Ávila-Batista, A.C., Rueda, F.J.M. \& Hauck Filho, N. (2019). Construcción y evidencias de validez de una escala abreviada de autoinforme para evaluar el autoritarismo. Acta Colombiana de Psicología, 22(1), 31-41. doi: http://www.dx.doi.org/10.14718/ACP.2019.22.1.3
\end{abstract}

Resumen

La agresión autoritaria, la sumisión autoritaria y el convencionalismo son creencias sociales o actitudes que forman parte del llamado autoritarismo. El presente estudio da cuenta de la construcción y análisis de las propiedades psicométricas de una escala abreviada para evaluar tendencias autoritarias, la Escala de Actitudes frente al Autoritarismo (ESCAUT). En total, participaron 786 brasileños adultos con edades entre los 18 y 75 años, el $70.7 \%$ eran militares. Inicialmente, se elaboraron 42 ítems para captar los principales tipos de creencias autoritarias, y los resultados de los análisis factoriales ordinales permitieron retener 20 ítems de moderados a altamente discriminatorios (cargas $>.50$ ) de dos factores latentes: autoritarismo moral y punitivo $(\alpha=.88)$ y autoritarismo sumiso $(\alpha=.89)$. Las curvas de información revelaron una amplia cobertura de la variable latente para ambas subescalas. Incluso, el instrumento fue capaz de discriminar participantes militares de no militares, con grandes efectos para las diferencias entre los grupos. En conclusión, la ESCAUT consiste en una escala abreviada de autoinforme que puede ser utilizada en la investigación de las principales actitudes autoritarias presentes en la población general. Al final se discuten las limitaciones del estudio.

Palabras clave: actitudes, prejuicio, grupos sociales.

\section{Construction and validity evidence of a brief self-report scale to assess authoritarianism}

\begin{abstract}
Authoritarian aggression, authoritarian submission, and conventionalism comprise beliefs or attitudes know as authoritarianism. In the present study, we report on the development and the test of the psychometric properties of a brief scale for the assessment of authoritarian tendencies, the Attitudes toward Authoritarianism Scale (ESCAUT). Participants in the study were 786 adults with ages ranging from 18 to 75 years, $70.7 \%$ military. An initial pool of 42 items written to capture the core features of authoritarianism was evaluated by specialists, and then answered by the participants. Results from ordinal factor analysis indicated 20 items with moderately to highly discriminative (factor loadings $>.50$ ) of two latent factors: Moral and punitive authoritarianism $(\alpha=.88)$, and submissive authoritarianism $(\alpha=.89)$. Test information curves revealed a broad coverage of the latent trait in both subscales. Moreover, the instrument could discriminate military from non-military participants, with large size differences. The ESCAUT is a brief self-report scale recommended for the assessment of the main features of authoritarianism in the general Latin American population. Limitations of the study are addressed.

Keywords: attitudes, prejudice, social groups.
\end{abstract}

\footnotetext{
* Calle Waldemar César da Silveira, 105, Vl. Cura D'Ars (SWIFT), Campinas - São Paulo CEP 13045-510. Teléfono: +55 19-37793371. fabian.rueda@usf.edu.br
} 


\title{
Construçáo e evidências de validade de uma escala abreviada de autorrelato para avaliar o autoritarismo
}

\begin{abstract}
Resumo
A agressão autoritária, a submissão autoritária e o convencionalismo são crenças sociais ou atitudes que fazem parte do chamado "autoritarismo". Este estudo apresenta a construção e análise das propriedades psicométricas de uma escala abreviada para avaliar tendências autoritárias, a Escala de Atitudes ante o Autoritarismo (Escaut). No total, participaram 786 brasileiros adultos, entre os 18 e 75 anos, $70.7 \%$ militares. Inicialmente, foram elaborados 42 itens para captar os principais tipos de crenças autoritárias, e os resultados das análises fatoriais ordinais permitiram reter 20 itens de moderados a altamente discriminatórios (cargas $>.50)$ de dois fatores latentes: autoritarismo moral e punitivo $(\alpha=.88)$ e autoritarismo submisso $(\alpha=.89)$. As curvas de informação revelaram uma ampla cobertura da variável latente para ambas as subescalas. Inclusive, o instrumento foi capaz de discriminar participantes militares de não militares, com grandes efeitos para as diferenças entre os grupos. Em conclusão, a Escaut consiste numa escala abreviada de autorrelato que pode ser utilizada na pesquisa das principais atitudes autoritárias presentes na população em geral. Ao final do artigo, são discutidas as limitações deste estudo.

Palavras-chave: atitudes, preconceito, grupos sociais.
\end{abstract}

\section{Introducción}

El autoritarismo es un constructo de constante interés en la psicología, con estudios desde fines del siglo XIX y con mayor énfasis a partir de mediados del siglo XX (Adorno, Frenkel-Brunswik, Levinson \& Sanford, 1950; Fromm, 1942). Este fenómeno ha sido analizado tanto desde la perspectiva de la interacción entre grupos sociales, como desde el abordaje de las diferencias individuales, incluyendo rasgos de personalidad constitutivos, creencias e influencias situacionales (Brussino \& Etchezahar, 2013). En este sentido, la evaluación del autoritarismo parte de la premisa de que existe una constelación de características que componen una "personalidad autoritaria" (Adorno et al., 1950), y, al estudiar esas características centrales, Altemeyer (1981) encontró tres grupos de rasgos correlacionados: la sumisión autoritaria -alto grado de complicidad con las autoridades, que son percibidas por el individuo como legítimas en la sociedad en la que vive-, la agresión autoritaria -agresividad dirigida a personas que son consideradas como merecedoras de sanciones por las autoridades establecidas $-\mathrm{y}$ el convencionalismo -alto grado de adhesión, aceptación y compromiso con normas sociales tradicionales apoyadas por las autoridades establecidas--. El autor afirmó que estas variables definen lo que se denominó como "autoritarismo de derecha" (right-wing authoritarianism), y el presente artículo da cuenta de la construcción de una escala breve de autoinforme para evaluar los aspectos centrales de esta definición.
En la literatura se ha encontrado que el autoritarismo se relaciona positivamente con una amplia gama de variables y eventos negativos en el ámbito interpersonal y social; en especial, con diversas formas de discriminación, incluyendo la intergrupal (Diáz-Lázaro, Castañeras, Ledesma \& Rand, 2014), la racial u homofóbica (Laythe, Finkel \& Kirkpatrick, 2001), la religiosa (Nuñes-Alacón, MorenoJiménez \& Moral-Toranzo, 2011), y la discriminación contra refugiados e inmigrantes (Gregurović, Kuti \& Župarić-Iljić, 2016), entre otras.

De acuerdo con Kossowska, Bukowski y Hiel (2008), esas manifestaciones de discriminación parecen ser más estimuladas tanto en situaciones de rabia como de tristeza. $Y$ se ha encontrado que individuos autoritarios tienden a adoptar actitudes rígidas y extremadamente tradicionales, siendo más propensos a defender papeles tradicionales para los géneros (Duncan, Peterson \& Winter, 1997), adoptar creencias religiosas fundamentalistas (Johnson, Labouff, Rowatt, Patock-Peckham \& Carlisle, 2012), contar con valores materialistas (Barros, Torres \& Pereira, 2009), dar menos importancia a los derechos humanos y a las libertades civiles (Moghaddam \& Vuksanovic, 1990), y ser más tolerantes frente a comportamientos abusivos y el uso de la tortura por parte de las autoridades (Larsson, Björklund \& Bäckström, 2012). También, se destaca que hombres autoritarios tienden a atribuirle culpa a las mujeres víctimas de violación (Manoussaki \& Veitch, 2015), a la vez que son más propensos a cometer agresión sexual (Walker, Rowe \& Quinsey, 1993). Por lo expuesto, el autoritarismo se presenta como una variable de impacto social en diversos ámbitos. 
Con respecto a los individuos, existen controversias sobre sí el autoritarismo constituye un factor de riesgo para problemas psicológicos o sí, por el contrario, representa un factor de protección. Una hipótesis presente en la literatura (Onraet \& van Hiel, 2014) es que individuos con una personalidad autoritaria serían resistentes y menos propensos a dejarse impactar por eventos agobiantes. $\mathrm{Si}$ se considera esa hipótesis como correcta, los individuos autoritarios serían capaces de afectar negativamente la vida de otras personas, sin ser afectados; sin embargo, los resultados no son conclusivos sobre ese punto de vista.

Aunque hay evidencias de que el autoritarismo puede funcionar como un amortiguador ("buffer") para algunos eventos de la vida (Van Hiel \& De Clercq, 2009), también las hay sobre que a largo plazo puede estar asociado a síntomas de depresión (Duriez, Klimstra, Luyckx, Beyers \& Soenens, 2012). Al respecto, una revisión de la literatura que discutió esa controversia señaló que, pese a que los individuos autoritarios interpreten el mundo como un lugar hostil, las evidencias no revelan resultados particularmente altos en ansiedad, depresión $\mathrm{u}$ otras variables internas del individuo (Onraet \& van Hiel, 2014). Es por esto que, aunque el problema no esté resuelto, ambas posibilidades señalan que el autoritarismo es un fenómeno psicológico que merece atención en el área de la evaluación clínica.

En este sentido, uno de los principales instrumentos de evaluación del autoritarismo es la escala de Right-Wing Authoritarianism (RWA; Altemeyer, 1981), que consiste en un inventario de autoinforme de 24 ítems que evalúan los tres rasgos de autoritarismo considerados como centrales por Altemeyer, es decir, la sumisión autoritaria, la agresión autoritaria y el convencionalismo. Este instrumento ofrece un resultado total, y su consistencia interna calculada por el coeficiente alfa es de .88 (Altemeyer, 1981). Actualmente, el instrumento cuenta con versiones adaptadas en diversos países, como Chile (Cárdenas \& Parra, 2011), España (Seoane \& Garzón, 1991), Suecia (Zakrisson, 2005), Rusia (McFarland, Ageyev \& Abalakina-Paap, 1992) y África del Sur (Duckitt, 1993), por citar algunos; y la escala tiene como mérito ser "la primera técnica moderna de medición psicológica aplicada al concepto" (Dusso, 2017, p. 244), siendo, tal vez, la más empleada en estudios del área (Dunwoody \& Funke, 2016). Además de esto, el instrumento ha sido bastante eficaz en el pronóstico tanto en estudios de la esfera social como política, así como en el estudio de actitudes de restricción a determinados grupos y minorías, y de la aceptación de tiranías gubernamentales (Gray \& Durrheim, 2013).

Pese a las cualidades relatadas sobre la RWA, esta escala también presenta algunas inconsistencias, especialmente en lo que respecta a su estructura factorial, ya que, a pesar de haber sido construida para evaluar tres conjuntos de rasgos, Altemeyer (1996) apenas encontró soluciones de uno y dos factores más ajustadas a los datos. El autor atribuyó esa divergencia a la dificultad de encontrar ítems que sean indicadores puros de apenas una de las dimensiones del autoritarismo, dada la multidimensionalidad de los comportamientos autoritarios. De hecho, estudios que trataron de replicar la propuesta de Altemeyer encontraron soluciones bastante diferentes; por ejemplo, con seis factores, en el estudio de Seoane y Garzón (1992).

Además de la complejidad y multidimensionalidad de los ítems, Mavor, Louis y Sibley (2010) sugirieron que dicha controversia se puede dar en función de estilos de respuesta, algo que no había sido tenido en cuenta en estudios del área; por lo que, después de controlar el sesgo de aquiescencia, encontraron resultados favorables para una estructura de tres factores. Asimismo, Funke (2005) recomendó una solución de tres factores en oposición a un resultado total -práctica recurrente en el área-, y demostró que la puntuación total del instrumento puede no estar relacionada con otras variables medidas, aunque los tres resultados factoriales sean predictivos de esas variables. Estas y otras limitaciones relacionadas con la dimensionalidad han inspirado varias revisiones del instrumento, así como la propuesta de versiones reducidas, con la eliminación de los ítems más problemáticos (Manganelli-Rattazzi, Bobbio \& Canova, 2007; Zakrisson, 2005).

Otro aspecto que debe ser considerado al analizar la RWA es el contenido de los ítems, ya que la escala fue construida tomando como base el contexto canadiense, uno muy diferente al de los países de América Latina con una historia de dictadura militar que ha dejado una herencia cultural específica. Posiblemente, en países como Brasil, Argentina, Chile, Colombia, Uruguay y Nicaragua, entre otros, las actitudes autoritarias adquieran una forma particular de expresión que las diferencie de otros países del hemisferio norte; y, teniendo esto en cuenta, por más que el uso de instrumentos de evaluación ya consolidados en otros países sea valioso por proporcionar comparaciones transculturales, también sería útil una escala elaborada para captar las particularidades del autoritarismo de la forma en que ocurre en América Latina.

Así, la elección de indicadores más apropiados para el contexto latinoamericano puede proporcionar una evaluación más refinada e informativa de los rasgos latentes que explican el apoyo de actitudes autoritarias, además de que, por más que sean contemplados los tres temas centrales del autoritarismo, la estructura factorial de una escala de actitudes autoritarias puede presentar un número de factores diferentes en el contexto latinoamericano. En este sentido, adicional a los 
beneficios de una medida nueva, sería interesante estudiar la estructura factorial de un inventario de actitudes compuesto por ítems escritos con el objetivo de captar de forma más amplia aspectos típicos de Brasil y de América Latina.

En virtud de eso, el objetivo de este estudio fue la construcción y el análisis de las propiedades psicométricas de una escala abreviada de evaluación de actitudes autoritarias, la Escala de Actitudes frente al Autoritarismo -llamada ESCAUT-. El instrumento fue elaborado con el fin de captar los principales aspectos conceptuales del autoritarismo, tal como fueron descritos por Altemeyer (1981, 1988, 1996) y por autores más recientes (Duckitt, Bizumic, Krauss \& Heled, 2010), a saber: la sumisión autoritaria, la agresión autoritaria y el convencionalismo.

Los ítems de la escala fueron elaborados teniendo en cuenta su aplicabilidad en el contexto latinoamericano, especialmente de Brasil, buscando maximizar el alcance latente del autoritarismo en estudios con muestras de ese tipo. Una ventaja es que la ESCAUT tuvo sus ítems elegidos con base en análisis factoriales adecuados a ítems de naturaleza ordinal, siguiendo recomendaciones de la literatura reciente (Asún, Rdz-Navarro \& Alvarado, 2015; Holgado-Tello, Chacón-Moscoso, Barbero-García \& VilaAbad, 2010). Incluso, el propósito fue la construcción de un instrumento cuyo número de ítems no representara un peso al ser considerada su inclusión en protocolos de investigación con múltiples medidas. Después de realizar el análisis psicométrico de la ESCAUT por medio de análisis factoriales, estimaciones de consistencia interna e inspección de curvas de información, se comparan los resultados entre un grupo de militares y otro de no militares. La hipótesis es que los militares puntuarían más alto en los factores del instrumento, teniendo en cuenta que el ambiente militar tiende a incentivar la adopción de actitudes autoritarias (Gatto, Dambrun, Kerbrat \& De Oliveira, 2009).

\section{Método}

\section{Diseño}

Se trata de un estudio de naturaleza transversal para la elaboración de una escala para evaluar el autoritarismo en adultos brasileños y comprobar la validez psicométrica de la escala.

\section{Participantes}

La muestra fue no probabilística, por conveniencia, compuesta por 786 adultos brasileños con edades de $18 \mathrm{a}$ 75 años $(M=33.04 ; D T=10.48)$, siendo $58 \%$ del sexo masculino. Con respecto a la escolaridad, los participantes contaban con postgrado (32.6\%), enseñanza universitaria completa $(25.3 \%)$, enseñanza universitaria incompleta $(24.2 \%)$ o enseñanza secundaria completa (17.9\%); mientras que, frente a su actuación profesional, la mayoría eran funcionarios públicos, principalmente militares estatales (70.7\%), seguidos de trabajadores tercerizados $(12.3 \%)$, y trabajadores del área comercial o industrial (7.3\%), el $9.7 \%$ no especificó el sector; y con respecto al lugar de residencia, el $81 \%$ vivía en Minas Gerais, el $7.3 \%$ en San Pablo, el $1.7 \%$ en Río de Janeiro, y el $10 \%$ en los demás estados brasileños.

\section{Instrumento}

Los ítems de la ESCAUT se crearon a partir de las definiciones de los tres grupos de rasgos descritos por Altemeyer-agresión autoritaria, sumisión autoritaria y convencionalismo-(1981, 1988, 1996), pero debe mencionarse una pequeña diferencia teórica: mientras que Altemeyer consideró que el autoritarismo es un conjunto de rasgos de personalidad, los ítems de la ESCAUT fueron creados para evaluar actitudes autoritarias. En otras palabras, al elaborar los ítems se dio menos énfasis a patrones estables del funcionamiento intraindividual, y se enfatizaron más las creencias sobre objetos o situaciones sociales, en concordancia con el concepto clásico de actitud (para una revisión, véase Kruglanski et al., 2015).

Además de esto, las concepciones modernas de autoritarismo han criticado la noción de "personalidad autoritaria" de Altemeyer, y en vez de ella, han considerado las dimensiones descritas para las actitudes ideológicas autoritarias (Duckitt et al., 2010). Una vez consideradas esas diferencias conceptuales, se procedió a escribir los ítems para evaluar los tres temas principales del autoritarismo, conservadurismo $\mathrm{y}$ tradicionalismo, comunes a los detallados por Altemeyer (1981) y la literatura más reciente (Duckit et al., 2010).

Teniendo en cuenta este modelo y el contexto brasileño, se elaboraron 49 ítems -tratando de mantener un equilibrio en el número de ítems para cada aspecto teórico-, 17 para la agresión autoritaria (p. ej.: "Para determinados crímenes, la única solución es la pena de muerte"), 17 la para sumisión autoritaria (p. ej.: "Las autoridades saben lo que es mejor para el pueblo") y 15 para el convencionalismo (p. ej.: "Personas que piensan diferente de las autoridades no deben ser estimuladas a expresar sus opiniones").

La pertinencia de esos ítems fue evaluada por seis doctores en psicología con experiencia en construcción de instrumentos -a los cuales se les envió el material vía electrónica con las definiciones de cada dimensión teórica-, con la finalidad de que indicaran en qué medida cada ítem era representativo del aspecto evaluado. También, se le 
solicitó a los jueces que realizaran un análisis semántico de los ítems.

Después de recibidas las respuestas de los jueces, se retiraron los ítems con concordancia inferior a $80 \%$ entre evaluadores, siguiendo las recomendaciones de Pasquali (2010). Además, de acuerdo con las sugerencias recibidas, se reformularon los ítems que tuvieron indicaciones de problemas en la redacción. Después de esta etapa, se obtuvo un total de 42 ítems que formaron la primera versión de la escala. Se hizo una escala de respuesta tipo Likert de cuatro puntos para cada ítem, siendo $1=$ totalmente en desacuerdo, 2 = en desacuerdo, $3=$ de acuerdo, y $4=$ totalmente de acuerdo.

\section{Procedimiento}

Inicialmente, tras la revisión aprobación del proyecto de investigación por parte de un Consejo de Ética en Investigación de una universidad brasileña, se solicitó autorización para la divulgación de la investigación en un órgano de Seguridad Pública del Estado de Minas Gerais, por ser este el local de trabajo de uno de los autores. También, se divulgó la ESCAUT en una red social (Facebook) como cuestionario online, y se envió un correo electrónico a la lista de contactos de los autores del estudio con el link que daba acceso al cuestionario online. Para acceder a las instituciones militares brasileñas se hizo una invitación de manera presencial para que participaran en la investigación. Todos los sujetos dieron su consentimiento para participar después de leer la información y las explicaciones sobre los objetivos del estudio. El tiempo promedio de respuesta de la escala fue de 15 minutos.

\section{Análisis de datos}

Primero, se evaluó la posibilidad de realizar análisis factorial exploratorio de los datos por medio del coeficiente Kaiser-Meyer-Olkin (KMO), que ofrece una medida de las correlaciones entre los ítems; posteriormente, se utilizó el test de Mardia para comprobar la normalidad multivariada de los datos; $y$, en seguida, se investigó la dimensionalidad de los datos por medio de tres técnicas diferentes: el análisis paralelo con permutación de valores (Timmerman \& Lorenzo-Seva, 2011), las correlaciones parciales mínimas (Velicer, 1976) y el método de Hull (Lorenzo-Seva, Timmerman \& Kaers, 2011).

Después de esto, se estimaron los parámetros de la solución más interpretable por medio de los cuadrados mínimos ponderados (Weighted Least Squares Mean and Variance-adjusted-WLSMV-), y a partir de la matriz de correlaciones policóricas entre las variables. Este estimador es robusto y especialmente recomendado para el análisis de ítems de naturaleza categórica ordinal, como lo es la escala tipo Likert de cuatro puntos (Asún et al., 2015). Teniendo en cuenta la propuesta de que la versión final de la ESCAUT fuese abreviada, solamente se retuvieron los ítems con discriminación entre moderada y alta, es decir, con cargas factoriales superiores a .50 en por lo menos uno de los factores.

También, se trató de evitar ítems con contenido muy redundante -con el fin de maximizar la cobertura del rasgo latente-, y se verificó la confiabilidad de las escalas compuestas por los ítems de cada factor tanto por medio de los coeficientes tradicionales de consistencia interna como con la curva de información de los ítems y del test. Estas curvas de información son más específicas que las estimaciones tradicionales de consistencia interna porque revelan la confianza del instrumento esperada para cada parte de rasgo latente.

Finalmente, se realizaron los análisis paramétricos $(t$ de Student) y no paramétricos (prueba Mann-WhitneyWilcoxon) de comparación de medias con el fin de investigar la capacidad del instrumento para discriminar individuos militares de no militares. Todos los análisis se realizaron utilizando los programas R, Factor (Lorenzo-Seva \& Ferrando, 2013) y Mplus (Muthén \& Muthén, 2017).

\section{Resultados}

Tras la inspección de la calidad de los datos, se realizó el análisis factorial, el cálculo de la consistencia interna, las curvas de informaciones y, finalmente, las comparaciones de los promedios entre el grupo de militares y no militares.

Inicialmente, se encontró un índice Kaiser-Meyer-Olkin de .95 , que sugiere un nivel suficiente de variancia común entre las variables para un análisis factorial. Asimismo, el test de Mardia reveló desvíos significativos $(p<.001)$ de la normalidad multivariada en el conjunto inicial de 42 ítems, es decir, otro motivo para el uso del estimador robusto elegido para los análisis factoriales, por lo que se procedió a la investigación de la dimensionalidad de los datos.

En particular, los métodos de retención no fueron unánimes, por lo cual se realizó la recomendación de tener en cuenta dos factores para el análisis paralelo con permutación de los datos y el método de los parciales medios mínimos, pero solo se consideró un factor para el método Hull. Además de esto, al considerar soluciones con más de un factor, muchos ítems presentaron variancia común baja o una tendencia a cargas cruzadas. Sin embargo, considerando que el propósito era que el instrumento fuera breve, se excluyeron estos ítems, y tan solo se escogieron los 20 mejores. 
Después de esto, se decidió probar el ajuste de soluciones factoriales exploratorios de uno y dos factores utilizando los índices RMSEA, CFI y TLI; y se encontró que mientras que el modelo de un factor presentaba un ajuste empobrecido de los datos $(\mathrm{RMSEA}=.091, \mathrm{CFI}=.890 \mathrm{y}$ $\mathrm{TLI}=.877)$, el modelo de dos factores presentaba un buen ajuste aproximado $(\mathrm{RMSEA}=.062, \mathrm{CFI}=.954$ y TLI $=$ .942). Cabe destacar que también se revisó una solución de tres factores, pero esa posibilidad fue descartada inmediatamente por no permitir interpretar el patrón de las cargas factoriales producidas. Teniendo esto en cuenta, los resultados favorecieron el modelo de dos factores, cuyos ítems, cargas factoriales y estimaciones de consistencia interna son presentados en la Tabla 1.

Como se puede observar en la Tabla 1, la solución factorial fue simple y con cargas cruzadas de baja magnitud $(<|.27|)$, siendo cada factor explicativo de 10 ítems, predominantemente. El Factor 1 explicó ítems relacionados con la agresión autoritaria y el convencionalismo descritos por Altemeyer (1981), por lo que fue denominado "Autoritarismo Moral y Punitivo". Mientras que el Factor 2 explicó ítems con contenido específico sobre sumisión a la autoridad, por lo que fue llamado "Autoritarismo Sumiso". Los dos factores se mostraron casi ortogonales, correlacionados en apenas $r=.05$, y las estimaciones de consistencia interna (alfa de Cronbach estándar), calculadas a partir de la naturaleza ordinal de las variables, fueron altas para las escalas formadas por los dos factores ( .88 y .89 para los factores 1 y 2 , respectivamente). Un análisis de consistencia interna con todos los ítems dio como resultado en un alfa aún más elevado, de .92.

La siguiente etapa consistió en profundizar aún más la confiabilidad del instrumento construido, por medio de las curvas de información de los dos factores. Estos análisis revelaron diferencias en el funcionamiento de cada factor, ya que el factor 'Autoritarismo Moral y Punitivo' presentó un nivel de precisión más alto para el intervalo entre $-1.50 \mathrm{y}+1.50$ de la escala theta -evidenciando que proporciona información sobre un amplio espectro latente arriba y abajo de la media de la población (estandarizada como 0.00)-, mientras que el factor "Autoritarismo Sumiso" presentó un mejor funcionamiento en regiones más bajas del rasgo latente -entre $-3.00 \mathrm{y}$ $0.00-$, pero con una amplia cobertura. Estos análisis sugieren que los ítems del factor "Autoritarismo Moral y Punitivo" evalúan aspectos más severos de actitudes autoritarias, en comparación con el factor "Autoritarismo Sumiso".

Por último, se realizó un test adicional de la capacidad discriminatoria del instrumento por medio de una comparación de medias entre militares y no militares, y, como los escores en los dos factores no presentaron distribución normal por la prueba de Shapiro-Wilks $(\mathrm{p}<.05)$, se tuvo la precaución

Tabla 1.

Resultados del Análisis Factorial con Estimador WLSMV

\begin{tabular}{|c|c|c|}
\hline & F1 & F2 \\
\hline 1. La sociedad actual se caracteriza por la inmoralidad y decadencia de valores tradicionales. & .78 & -.05 \\
\hline 2. Para determinados crímenes, la única solución es la pena de muerte. & .70 & -.23 \\
\hline 3. Niños que no obedecen a los padres o profesores deben ser castigados. & 69 & -.04 \\
\hline 4. La educación moderna está tornando a los adolescentes rebeldes, perjudicando los buenos valores. & .65 & .02 \\
\hline 5. Consumidores de drogas deben ser castigados, y no recuperados. & .64 & .04 \\
\hline 6. Si la moral y las buenas costumbres no son seguidas, la sociedad se tornará un caos. & .64 & .15 \\
\hline 7. Las personas solo aprenden lo que es correcto si son castigadas con rigor. & .58 & .11 \\
\hline 8. Los valores familiares tradicionales deben ser mantenidos a cualquier costo. & .57 & .27 \\
\hline 9. La sociedad debe castigar severamente a quien cometa cualquier tipo de delito. & .53 & .00 \\
\hline $\begin{array}{l}\text { 10. Personas que no siguen las normas establecidas deben ser castigadas para que sirvan de ejemplo y sus } \\
\text { actos no se repitan. }\end{array}$ & .52 & .17 \\
\hline 11. Los líderes deben ser seguidos sin cuestionamientos. & -.15 & .93 \\
\hline 12. Lo que los religiosos dicen debe ser seguido por todos. & -.01 & .75 \\
\hline 13. Enseñamientos religiosas fueron hechos para ser seguidos, y no debatidos. & .01 & .74 \\
\hline 14. Las normas establecidas no deben ser cuestionadas. & .04 & .70 \\
\hline 15. Las autoridades saben lo que es mejor para el pueblo. & -.01 & 68 \\
\hline 16. La sociedad precisa de personas sumisas, que no cuestionen lo que está establecido. & .02 & .66 \\
\hline 17. Personas que piensan diferente de las autoridades no deben ser estimuladas a expresar sus opiniones. & .00 & .65 \\
\hline 18. Las personas deben seguir los principios morales dictados por las autoridades. & .07 & .56 \\
\hline 19. Las personas deben cumplir lo que las autoridades exigen. & .07 & .53 \\
\hline 20. Solo las personas obedientes a Dios pueden ser consideradas buenas. & .13 & .53 \\
\hline Coeficiente alfa & .88 & .89 \\
\hline Confiabilidad de los factores rotados & .90 & .91 \\
\hline
\end{tabular}



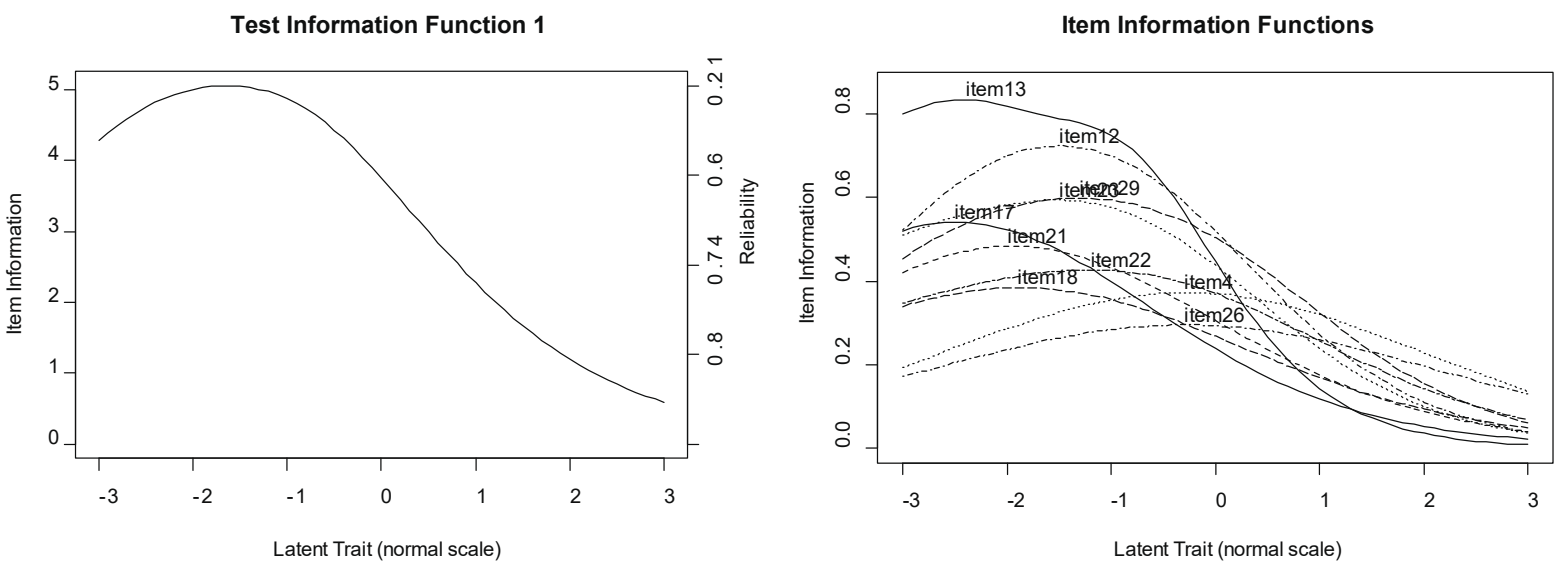

Figura 1. Curvas de información de la escala y de los ítems de Autoritarismo Moral y Punitivo.
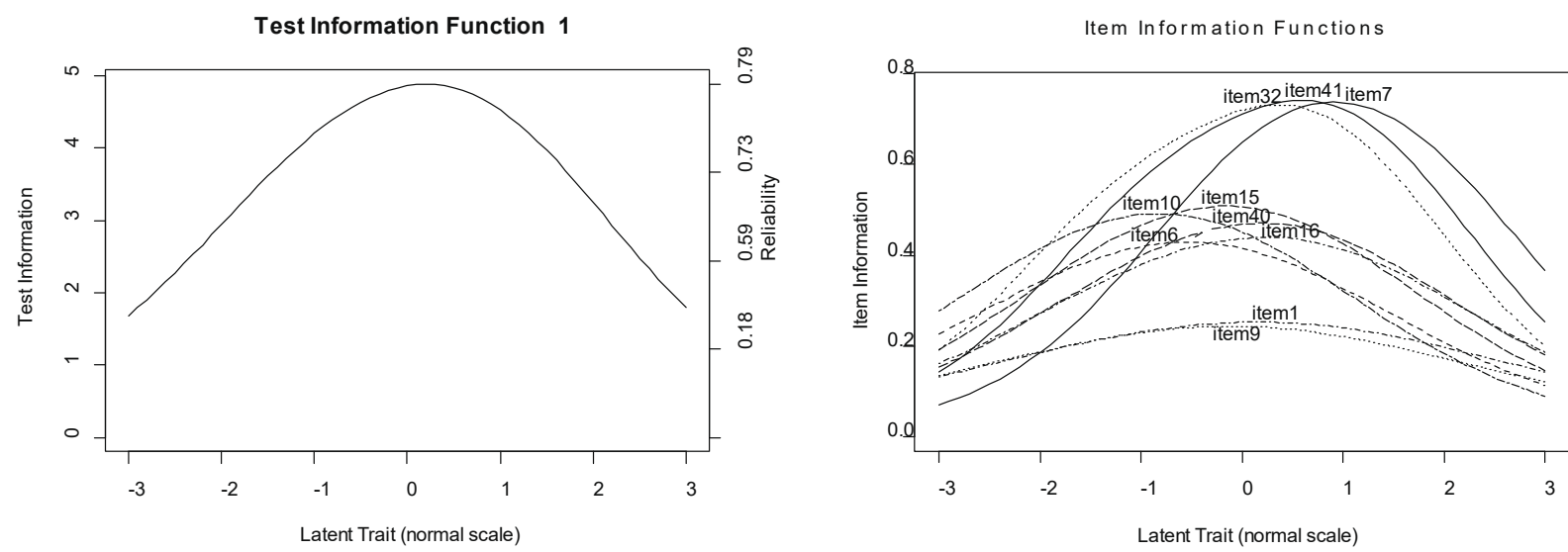

Figura 2. Curvas de información de la escala y de los ítems de Autoritarismo Sumiso.

de averiguar diferencias entre los grupos con una prueba no paramétrica. Como resultado, todas las comparaciones revelaron un efecto de gran magnitud, siendo que los militares presentaban un puntaje significativamente más alto en el factor "Autoritarismo Moral y Punitivo" (W = 120750, $p<.001, d=1.11$ ), en el factor "Autoritarismo Sumiso" $(\mathrm{W}=116320, p<.001, d=.90), \mathrm{y}$ también en la puntuación total del instrumento $(\mathrm{W}=123850, p<.001, d=1.17)$.

\section{Discusión}

El objetivo del presente artículo fue dar cuenta de la construcción y análisis de las propiedades psicométricas de una escala abreviada de autoinforme para evaluar los componentes centrales de las actitudes autoritarias (Altemeyer, 1981, 1988, 1996). Los análisis factoriales ordinales de los ítems elaborados permitieron llegar a una solución interpretable de dos factores casi ortogonales, cada uno con 10 ítems.
El primer factor, denominado "Autoritarismo Moral y Punitivo", explicó ítems más característicos de los aspectos de agresión autoritaria y, parcialmente, del convencionalismo, tal como fueron descritos por Altemeyer (1981), lo cual significa que individuos que puntúan alto en este factor tienden a manifestar excesiva adhesión a las normas y valores tradicionales, además de hostilidad dirigida a grupos o individuos que se desvían de esas normas. Así, es posible que este factor sea predictivo de discriminación, xenofobia y simpatía por el uso de la violencia por parte de las autoridades, y puede también relacionarse con altas puntuaciones en inestabilidad emocional y bajos puntajes en amabilidad. Estas posibles asociaciones, no obstante, todavía requieren de nuevos estudios empíricos.

A su vez, el segundo factor, denominado "Autoritarismo Sumiso", se puede interpretar como una combinación entre la sumisión autoritaria y el convencionalismo de Altemeyer, es decir, que personas con altos puntajes en este factor tienden a manifestar una obediencia inflexible a las autoridades, y son vistas como poseedoras de una 
conducta moralmente correcta en todas las circunstancias. Ese factor puede estar relacionado con el fundamentalismo religioso y, en términos de personalidad, con rasgos como responsabilidad e inestabilidad emocional.

Por otra parte, el conjunto final de los 20 ítems de la escala presentó excelentes propiedades psicométricas, ya que ambos factores tuvieron consistencia interna próxima a .90 , siendo el alfa de la escala total equivalente a .92 ; valores superiores a los encontrados para la RWA, que fue de .88 en Altemeyer (1981) y de .72 en Cárdenas y Parra (2011), por ejemplo. Asimismo, vale destacar que la consistencia interna de la ESCAUT también se mostró superior a la encontrada en las versiones reducidas de la RWA - elaboradas a partir de los mejores ítems de la escala- (por ejemplo, de .72 a .80 en Zakrisson [2005]; y de .85 en Manganelli-Rattazzi et al. [2007]).

Adicionalmente, el análisis de las curvas de información reveló que la elevada consistencia interna no fue obtenida al costo de la inclusión de ítems redundantes, sino que, al contrario, se priorizó mantener ítems que cubrieran un amplio espectro de las actitudes autoritarias. Aunque se considere que eso ocurrió de forma más satisfactoria en el Factor 1 (cuyo pico de información se ajusta más a la media de la población,--véase Figura 1-), el instrumento puede ser considerado como una herramienta capaz de proporcionar una evaluación rápida y bastante informativa de las actitudes autoritarias. Por esto, se considera la ESCAUT como una herramienta útil para la investigación en diversos grupos sociales, desde individuos poco autoritarios a personas con características autoritarias prominentes.

Además de esto, los resultados también sostienen la capacidad del instrumento para discriminar grupos de individuos con varios niveles de autoritarismo. Específicamente, para esta investigación se eligió una muestra de militares para comparar con los demás participantes, $\mathrm{y}$, como se esperaba, hubo diferencias muy grandes entre los grupos, en donde los resultados de los militares fueron más altos que los de los no militares.

Aunque los resultados de esta investigación sustenten la validez de la ESCAUT, estos deben ser vistos con cautela, ya que las diferencias de media aquí expuestas no deben ser tomadas como evidencia de que los militares siempre van a manifestar actitudes más autoritarias que los no militares. Apenas para ilustrar, al comparar un grupo de policías clasificados como "innovadores" y otro denominado "tradicionales", Carlson, Thayer y Germann (1971) comprobaron que solo el segundo grupo puntuó más alto que una muestra de estudiantes en una escala de autoritarismo. De este modo, aunque el ambiente de trabajo de militares y policías inspire respeto a la autoridad y apoyo a actitudes autoritarias (Gatto et al., 2009; Worden, 1995), el nivel de autoritarismo en esos grupos puede depender de otros aspectos, incluyendo su función dentro de la organización (Perrott \& Taylor, 1995; Rubinstein, 2006). En suma, la creencia de que militares y policías son siempre autoritarios y agresivos no necesariamente se sustenta empíricamente, y esto es algo que no debería ser generalizado para otros grupos diferentes a los de la muestra aquí empleada (Laguna, Linn, Ward \& Rupslaukyte, 2010).

Además de lo mencionado, también debe tenerse en cuenta que la propuesta teórica de Altemeyer (1981) sugiere que existen tres dimensiones teóricas del autoritarismo: la sumisión autoritaria, la agresión autoritaria y el convencionalismo; sin embargo, el instrumento elaborado por dicho autor para evaluar tales características no presenta una estructura factorial consistente. Para explicar esta limitación en la replicabilidad factorial, se han considerado varias hipótesis en la literatura, como, por ejemplo, que algunos ítems poseen propiedades psicométricas pobres (Manganelli-Rattazzi et al., 2007; Zakrisson, 2005) o que el instrumento es una combinación de estilos de respuesta (Mavor et al., 2010). En este mismo orden, en el presente estudio tampoco se encontraron esas tres dimensiones, sino tan solo dos, resultado que también fue encontrado por Altemeyer (1996) para su escala RWA.

De forma general, estos hallazgos cuestionan la legitimidad de las tres dimensiones teóricas de Altemeyer como si fueran factores distintos que actúan como causas latentes y que explican los comportamientos de los individuos. Por lo tanto, es posible que solo dos dimensiones sean suficientes para explicar los aspectos más centrales del autoritarismo, a saber, el Autoritarismo Moral y Punitivo, y el Autoritarismo Sumiso. Para investigar este aspecto, estudios futuros podrían comparar la consistencia y la interpretabilidad del patrón de correlatos externos de dos versus tres factores del autoritarismo, en el contexto de una variedad de escalas de autoritarismo o aspectos relacionados.

Finalmente, a pesar de confirmar el uso de la ESCAUT para fines de investigación, el presente estudio posee algunas limitaciones, como que no hubo ningún control en los tipos de respuesta al investigarse la estructura interna del instrumento. Al respecto, como destacan van Vaerenbergh y Thomas (2013), tendencias a respuestas extremas y con aquiescencia pueden impactar la correlación de los ítems y, de esta manera, producir sesgos en los parámetros de una solución factorial; por lo que sería importante que estudios futuros evaluaran el impacto de respuestas extremas y aquiescentes en la estructura factorial y en la confiabilidad de la ESCAUT. Adicionalmente, otra limitación se refiere a un posible funcionamiento diferencial de los ítems según el sexo de los participantes, ya que las comparaciones de media que fueron efectuadas para estimar las diferencias 
entre militares y no militares asumen que los ítems de la ESCAUT funcionan de manera equivalente (es decir, que poseen la misma carga factorial e interceptación) tanto para hombres como para mujeres. En este sentido, sería importante analizar dicho aspecto en futuras investigaciones, lo que permitiría incluso perfeccionar el instrumento, tal vez, al identificar ítems con funcionamiento diferencial que deban ser suprimidos al comparar los grupos.

En conclusión, la ESCAUT demuestra ser una escala abreviada de autoinforme valida y confiable que puede ser utilizada en la investigación de las principales actitudes autoritarias presentes en la población en general. Se espera que esta escala pueda servir para la comprensión de fenómenos actuales relevantes tanto individuales como grupales, y que, teniendo en cuenta las similitudes históricas del contexto brasileño con otros países latinoamericanos, la traducción de la escala al español puede ser útil para muchos otros investigadores interesados en el estudio del autoritarismo, principalmente en momentos como el actual, en donde el conservadurismo y la política de derecha vuelven a destacar en diferentes espacios y contextos.

\section{Referencias}

Adorno, T. H., Frenkel-Brunswik, E., Levinson, D., \& Sanford, N. (1950). The Authoritarian Personality. New York: Harper.

Altemeyer, B. (1981). Right-Wing Authoritarianism. Winnipeg: University of Manitoba Press.

Altemeyer, B. (1988). Enemies of freedom: Understanding Right-Wing Authoritarianism. San Diego, CA: Jossey-Bass Inc., Publishers.

Altemeyer, B. (1996). The Authoritarian Specter. Cambridge: Harvard University Press.

Asún, R. A., Rdz-Navarro, K., \& Alvarado, J. M. (2015). Developing Multidimensional Likert Scales Using Item Factor Analysis: The Case of Four-point Items. Sociological Methods \& Research, 45(8), 744-749. doi: https://doi. org/10.1177/0049124114566716

Barros, T. S., Torres, A. R. R., \& Pereira, C. (2009). Psico-USF (Vol. 14). Universidade São Francisco. Recuperado de http://pepsic.bvsalud.org/scielo.php?script=sci_arttext\&pid $=\mathrm{S} 1413-82712009000100006$

Brussino, S., \& Etchezahar, E. (2013). Psychological perspectives in the study of authoritarianism. Journal of Alternative Perspectives in the Social Sciences, 5(3), 495-521. Recuperado de http://www.academia.edu/5869902/Psychological perspectives_in_the_study_of_authoritarianism

Cárdenas, M., \& Parra, L. (2011). Adaptación y validación de la Versión Abreviada de la Escala de Autoritarismos de Derechas (RWA) en una muestra chilena. Revista de
Psicología, 19(1), 61-79. doi: https://doi.org/10.5354/07190581.2010.17098

Carlson, H., Thayer, R. E., \& Germann, A. C. (1971). Social Attitudes and Personality Differences among Members of Two Kinds of Police Departments (Innovative vs. Traditional) and Students. The Journal of Criminal Law, Criminology, and Police Science, 62(4), 564. doi: https://doi. org/10.2307/1141714

Diáz-Lázaro, C. M., Castañeras, C., Ledesma, R. D., S., V., \& Rand, A. (2014). Right-Wing Authoritarianism, social dominance orientation, empathy, and materialistic value orientation as predictors of intergroup prejudice in Argentina. Salud \& Sociedad, 5(3), 282-297. doi: https://doi. org/10.22199/S07187475.5014.0003.00004

Duckitt, J. (1993). Right-Wing Authoritarianism Among White South African Students: Its Measurement and Correlates. The Journal of Social Psychology, 133(4), 553-563. doi: https://doi.org/10.1080/00224545.1993.9712181

Duckitt, J., Bizumic, B., Krauss, S. W., \& Heled, E. (2010). A Tripartite Approach to Right-Wing Authoritarianism: The Authoritarianism-Conservatism-Traditionalism Model. Political Psychology, 31(5), 685-715. doi: https://doi. org/10.1111/j.1467-9221.2010.00781.x

Duncan, L. E., Peterson, B. E., \& Winter, D. G. (1997). Authoritarianism and Gender Roles: Toward a Psychological Analysis of Hegemonic Relationships. Personality and Social Psychology Bulletin, 23(1), 41-49. doi: https://doi. org/10.1177/0146167297231005

Dunwoody, P. T., \& Funke, F. (2016). The Aggression-Submission-Conventionalism Scale: Testing a new three factor measure of authoritarianism. Journal of Social and Political Psychology, 4(2), 571-600. doi: https://doi.org/10.5964/ jspp.v4i2.168

Duriez, B., Klimstra, T. A., Luyckx, K., Beyers, W., \& Soenens, B. (2012). Right-Wing Authoritarianism: Protective Factor Against or Risk Factor for Depression? European Journal of Personality, 26(5), 536-549. doi: https://doi.org/10.1002/ per. 853

Dusso, A. (2017). Race and Right-Wing Authoritarianism: How Scoring High in Authoritarianism Does Not Necessarily Lead to Support for Right-Wing Candidates. Social Science Quarterly, 98(1), 244-260. doi: https://doi.org/10.1111/ ssqu. 12302

Fromm, E. (1942). The fear of freedom. London: Routledge.

Funke, F. (2005). The Dimensionality of Right-Wing Authoritarianism: Lessons from the Dilemma between Theory and Measurement. Political Psychology, 26(2), 195-218. doi: https://doi.org/10.1111/j.1467-9221.2005.00415.x

Gatto, J., Dambrun, M., Kerbrat, C., \& De Oliveira, P. (2009). Prejudice in the police: On the processes underlying the effects of selection and group socialisation. European Journal of Social Psychology, 40, 252-269. doi: https://doi. org/10.1002/ejsp.617 
Gray, D., \& Durrheim, K. (2013). Collective Rights and Personal Freedoms: A Discursive Analysis of Participant Accounts of Authoritarianism. Political Psychology, 34(4), 631-648. doi: https://doi.org/10.1111/j.1467-9221.2012.00932.x

Gregurović, M., Kuti, S., \& Župarić-Iljić, D. (2016). Attitudes towards Immigrant Workers and Asylum Seekers in Eastern Croatia: Dimensions, Determinants and Differences. Migracijske I Etničke Teme, 32(1), 91-122. doi: https://doi. org/10.11567/met.32.1.4

Holgado-Tello, F. P., Chacón-Moscoso, S., Barbero-García, I., \& Vila-Abad, E. (2010). Polychoric versus Pearson correlations in exploratory and confirmatory factor analysis of ordinal variables. Quality \& Quantity, 44(1), 153-166. doi: https://doi.org/10.1007/s11135-008-9190-y

Johnson, M. K., Labouff, J. P., Rowatt, W. C., Patock-Peckham, J. A., \& Carlisle, R. D. (2012). Facets of Right-Wing Authoritarianism Mediate the Relationship Between Religious Fundamentalism and Attitudes Toward Arabs and African Americans. Journal for the Scientific Study of Religion, 51(1), 128-142. doi: https://doi.org/10.1111/j.1468-5 906.2011.01622.x

Kruglanski, A. W., Jasko, K., Chernikova, M., Milyavsky, M., Babush, M., Baldner, C., \& Pierro, A. (2015). The rocky road from attitudes to behaviors: Charting the goal systemic course of actions. Psychological Review, 122(4), 598-620. doi: https://doi.org/10.1037/a0039541

Kossowska, M., Bukowski, M., \& Hiel, A. Van. (2008). The impact of submissive versus dominant authoritarianism and negative emotions on prejudice. Personality and Individual Differences, 45(8), 744-749. doi: https://doi.org/10.1016/j. paid.2008.07.022

Laguna, L., Linn, A., Ward, K., \& Rupslaukyte, R. (2010). An Examination of Authoritarian Personality Traits among Police Officers: The Role of Experience. Journal of Police and Criminal Psychology, 25(2), 99-104. doi: https://doi. org/10.1007/s11896-009-9060-0

Larsson, M. R., Björklund, F., \& Bäckström, M. (2012). Rightwing authoritarianism is a risk factor of torture-like abuse, but so is social dominance orientation. Personality and Individual Differences, 53(7), 927-929. doi: https://doi. org/10.1016/j.paid.2012.06.015

Laythe, B., Finkel, D., \& Kirkpatrick, L. A. (2001). Predicting Prejudice from Religious Fundamentalism and Right-Wing Authoritarianism: A Multiple-Regression Approach. Journal for the Scientific Study of Religion, 40(1), 1-10. doi: https://doi.org/10.1111/0021-8294.00033

Lorenzo-Seva, U., \& Ferrando, P. J. (2013). FACTOR 9.2: A Comprehensive Program for Fitting Exploratory and Semiconfirmatory Factor Analysis and IRT Models. Applied Psychological Measurement, 37(6), 497-498. doi: https://doi. org/10.1177/0146621613487794

Lorenzo-Seva, U., Timmerman, M. E., \& Kiers, H. A. L. (2011). The Hull Method for Selecting the Number of Common
Factors. Multivariate Behavioral Research, 46(2), 340-364. doi: https://doi.org/10.1080/00273171.2011.564527

Manganelli Rattazzi, A. M., Bobbio, A., \& Canova, L. (2007). A short version of the Right-Wing Authoritarianism (RWA) Scale. Personality and Individual Differences, 43(5), 12231234. doi: https://doi.org/10.1016/j.paid.2007.03.013

Manoussaki, K., \& Veitch, F. (2015). Ambivalent Sexism, Right Wing Authoritarianism and Rape Myth Acceptance in Scotland. International Journal of Gender \& Women's Studies, 3(1). doi: https://doi.org/10.15640/ijgws.v3n1a9

Mavor, K. I., Louis, W. R., \& Sibley, C. G. (2010). A bias-corrected exploratory and confirmatory factor analysis of right-wing authoritarianism: Support for a three-factor structure. Personality and Individual Differences, 48(1), 28-33. doi: https://doi.org/10.1016/j.paid.2009.08.006

McFarland, S. G., Ageyev, V. S., \& Abalakina-Paap, M. A. (1992). Authoritarianism in the former Soviet Union. Journal of Personality and Social Psychology, 63(6), 10041010. doi: https://doi.org/10.1037/0022-3514.63.6.1004

Moghaddam, F. M., \& Vuksanovic, V. (1990). Attitudes and Behavior Toward Human Rights Across Different Contexts the Role of Right-Wing Authoritarianism, Political Ideology, and Religiosity. International Journal of Psychology, 25(2), 455-474. doi: https://doi.org/10.1080/00207599008247877

Muthén, L. K., \& Muthén, B. O. (2017). Mplus user's guide. (7. ${ }^{a}$ Ed.). Los Angeles: Muthén \& Muthén.

Nuñes-Alacón, M., Moreno-Jiménez, M. P., \& Moral-Toranzo. (2011). Modelo causal del prejuicio religioso. Anales de Psicologia, 27(3), 852-861.

Onraet, E., \& van Hiel, A. (2014). Are Right-Wing Adherents Mentally Troubled? Recent Insights on the Relationship of Right-Wing Attitudes With Threat and Psychological IllBeing. Current Directions in Psychological Science, 23(1), 35-40. doi: https://doi.org/10.1177/0963721413514249

Pasquali, L. (2010). Instrumentação psicológica: fundamentos e práticas. Porto Alegre: Artmed.

Perrott, S. B., \& Taylor, D. M. (1995). Attitudinal Differences Between Police Constables and Their Supervisors. Criminal Justice and Behavior, 22(3), 326-339. doi: https://doi. org/10.1177/0093854895022003009

Rubinstein, G. (2006). Authoritarianism Among Border Police Officers, Career Soldiers, and Airport Security Guards at the Israeli Border. The Journal of Social Psychology, 146(6), 751-761. doi: https://doi.org/10.3200/SOCP.146.6.751-761

Seoane, J., \& Garzón, A. (1992). Creencias sociales contemporáneas, autoritarismo y humanismo. Psicología Política, 5, 27-52. Recuperado de https://dialnet.unirioja.es/servlet/ articulo? codigo $=2826354$

Timmerman, M. E., \& Lorenzo-Seva, U. (2011). Dimensionality assessment of ordered polytomous items with parallel analysis. Psychological Methods, 16(2), 209-20. doi: https://doi.org/10.1037/a0023353 
Van Hiel, A., \& De Clercq, B. (2009). Authoritarianism is good for you: Right-wing authoritarianism as a buffering factor for mental distress. European Journal of Personality, 23(1), 33-50. doi: https://doi.org/10.1002/per.702

Velicer, W. (1976). Determining the number of components from the matrix of partial correlations. Psychometrika, 41(3), 321-327. doi: https://doi.org/10.1007/BF02293557

Walker, W. D., Rowe, R. C., \& Quinsey, V. L. (1993). Authoritarianism and sexual aggression. Journal of Personality and Social Psychology, 65(5), 1036-1045. doi: https://doi. org/10.1037/0022-3514.65.5.1036
Worden, R. E. (1995). Police officers' belief systems: a framework for analysis. American Journal of Police, 14(1), 4981. doi: https://doi.org/10.1108/07358549510152979

Zakrisson, I. (2005). Construction of a short version of the Right-Wing Authoritarianism (RWA) scale. Personality and Individual Differences, 39(5), 863-872. doi: https://doi. org/10.1016/j.paid.2005.02.026 